\title{
Influence of Gold Nanoparticles on the Kinetics of $\alpha$-Synuclein Aggregation
}

\author{
Yanina D. Álvarez, ${ }^{\dagger, \ddagger} \|$ Jonathan A. Fauerbach, ${ }^{\S, \nabla, \|}$ Jésica V. Pellegrotti, $^{\dagger, \ddagger}$ Thomas M. Jovin, $^{\#}$ \\ Elizabeth A. Jares-Erijman, ${ }^{\S, \nabla, \perp}$ and Fernando D. Stefani ${ }^{*}, \dagger, \ddagger$
}

${ }^{\dagger}$ Departamento de Física, Facultad de Ciencias Exactas y Naturales (FCEN), Universidad de Buenos Aires (UBA), Buenos Aires, Argentina.

${ }^{\ddagger}$ Instituto de Física de Buenos Aires (IFIBA), Consejo Nacional de Investigaciones Científicas y Técnicas (CONICET), Buenos Aires, Argentina

${ }^{\S}$ Departamento de Química Orgánica, Facultad de Ciencias Exactas y Naturales (FCEN), Universidad de Buenos Aires (UBA), , Buenos Aires, Argentina

${ }^{\nabla}$ Centro de Investigación en Hidratos De Carbono (CIHIDECAR), Consejo Nacional de Investigaciones Científicas y Técnicas (CONICET), Buenos Aires, Argentina

"Laboratory for Cellular Dynamics, Max Planck Institute for Biophysical Chemistry, Goettingen, Germany

ABSTRACT: $\alpha$-synuclein (AS) is a small (140 amino acids), abundant presynaptic protein, which lacks a unique secondary structure in aqueous solution. Amyloid aggregates of AS in dopaminergic neurons of the midbrain are the hallmark of Parkinson's disease (PD). The process of aggregation involves a series of complex structural transitions from innocuous monomeric AS to oligomeric, presumably neurotoxic, forms and finally to fibril formation. Despite its potential importance for understanding PD pathobiology and devising rational, targeted therapeutic strategies, the details of the aggregation process remain largely unknown. Methodologies and reagents capable of controlling the aggregation kinetics are essential tools for the investigation of the molecular mechanisms of amyloid diseases. In this work, we investigated the influence of citratecapped gold nanoparticles on the aggregation kinetics of AS using a fluorescent probe (MFC) sensitive to the polarity of the molecular microenvironment via excited state intramolecular proton transfer (ESIPT). The particular effects on the half time, nucleation time, and growth rate were ascertained. Gold nanoparticles produced a strong acceleration of protein aggregation with an influence on both the nucleation and growth phases of the overall mechanism. The effects were dependent on the size and concentration of the nanoparticles, being strongest for nanoparticles $10 \mathrm{~nm}$ in diameter, which produced a 3-fold increase in the overall aggregation rate at concentrations as low as $20 \mathrm{nM}$.

KEYWORDS: Gold nanoparticles, amyloid proteins, amyloid aggregation, Parkinson
A myloid protein aggregation is related to about 20 diseases including Alzheimer's disease, Parkinson's disease (PD), type II diabetes, and spongiform encephalopathies. Common to all these conditions is a complex aggregation of proteins that are normally soluble. The mechanism involves the formation of various oligomers and intermediate supramolecular assemblies, which subsequently associate into insoluble fibrils that accumulate in the affected cells, tissues, and organs. The protein $\alpha$-synuclein (AS) is a small protein of 140 amino acids whose amyloid aggregation has been firmly linked to the pathogenesis of PD by numerous observations. ${ }^{1}$ A hallmark lesion of PD is the presence of spherical protein inclusions, called Lewy bodies, in the cytoplasm of dopaminergic neurons of the substantia nigra in the midbrain. Lewy bodies consist of a dense core surrounded by a halo of radial fibrils and are constituted of various proteins, the most abundant of which is
AS. The fibrils observed in PD are linear rods $5-10 \mathrm{~nm}$ in diameter and several micrometers long and similar to those arising in the other amyloid diseases. Cytotoxicity studies have indicated that oligomeric AS intermediates produced at early stages of the aggregation process are the most toxic species, ${ }^{2,3}$ although the participation of fibrils, particularly in cell-cell transfer of AS, ${ }^{4,5}$ has not been excluded. Whereas the structural details of fibrils have been elucidated in detail, ${ }^{6}$ much less is known about the nature of a number of intermediate oligomeric and supramolecular structures of AS present during the early stages of aggregation. ${ }^{7,8}$ Direct control of the oligomerization and fibrillization processes may enable effective therapeutical

Received: September 18, 2013

Revised: November 8, 2013

Published: November 12, 2013 

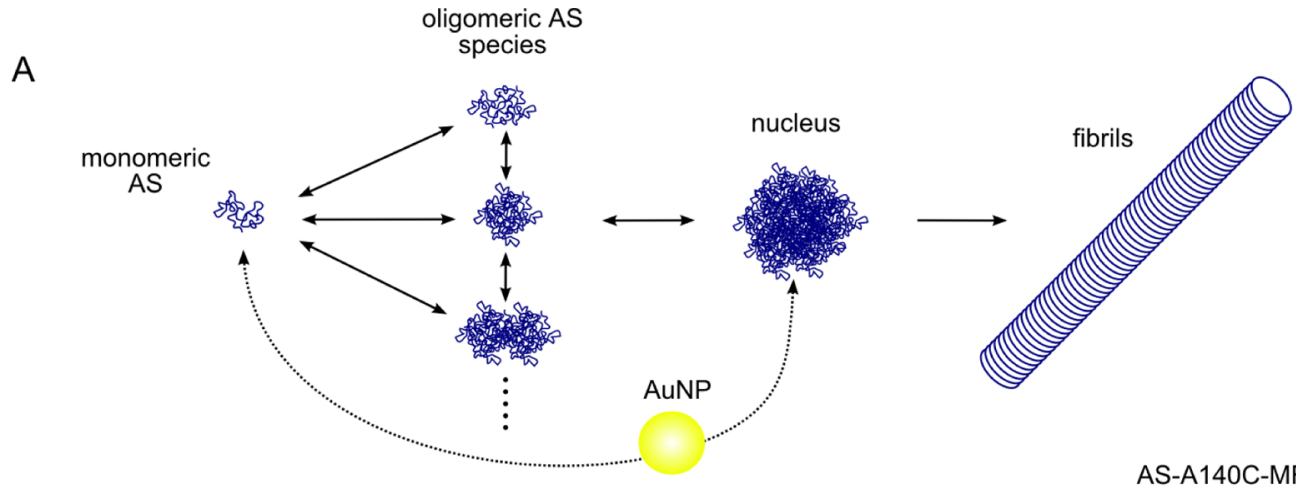

B

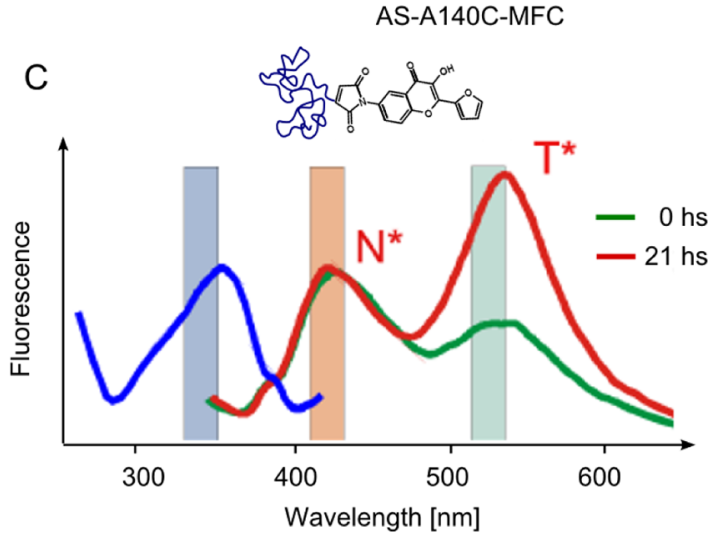

Figure 1. (A) Schematic of the aggregation process of $\alpha$-synuclein (AS). Gold nanoparticles (AuNPs) may interact with monomeric and/or oligomeric AS during the process of nucleation. (B) Diagram of the sigmoidal kinetics corresponding to nucleation followed by fast, autocatalytic growth. (C) The MFC fluorophore, which exhibits a strong environment-sensitive ESIPT response giving rise to changes in its dual emission, was covalently linked to a point mutant of AS with a cysteine residue at the C-terminus (AS-A140C). The AS-140C-MFC enables the sensitive monitoring of the aggregation process: fluorescence excitation spectrum (blue), emission spectra at start (green) and upon completion (red) of an aggregation assay of AS wild-type at $37^{\circ} \mathrm{C} .{ }^{34}$ Vertical bands indicate the spectral ranges used for excitation and detection of fluorescence.

strategies so far lacking for PD and related neurodegenerative diseases.

An attractive approach to gain control over the aggregation process of amyloid proteins is the use of nanoparticles as artificial chaperones to promote and control the formation of amyloidogenic as well as inhibitory structures. Exploration of this strategy is of recent origin and only a few reports can be found in the literature. Nanoparticles of different kinds (copolymeric, inorganic, and carbon nanotubes) enhance the rate of fibril formation of human 2-microglobulin $(\beta 2 \mathrm{~m})$ by promoting nucleation on the surfaces of the nanoparticles. ${ }^{9}$ The aggregation of amyloid- $\beta$ peptide has been modified by means of polymeric nanoparticles. ${ }^{10-13}$ The only report to our knowledge of nanoparticles influencing the aggregation of AS involved $\mathrm{CdSe} / \mathrm{ZnS}$ quantum dots (QDs) functionalized with streptavidin and linked to AS via a biotinylated mutant residue A90C. ${ }^{14}$ The aggregation of AS was strongly accelerated by subnanomolar concentrations of nanoparticles. Although the outstanding fluorescent properties of quantum dots have been extensively used in cellular studies, as, for example, in this system, ${ }^{15}$ the cytotoxicity of the semiconductor materials can pose a problem for application on live cells or in vivo experimentation. ${ }^{16}$ On the other hand, gold nanoparticles (AuNPs) possess demonstrated biocompatibility, ${ }^{17-21}$ and a large number of protocols exist for the controlled functionalization of their surface. ${ }^{19,21}$ Recently, the equilibrium association constants for complexes of AS and AuNPs at low temperature $\left(4{ }^{\circ} \mathrm{C}\right)$ were determined, obtaining a larger value for larger $(90$ $\mathrm{nm}$ diameter) than for smaller $(20 \mathrm{~nm})$ particles. $^{22}$ Their use as potential prion drugs has also been investigated, ${ }^{23}$ as well their capacity to generate local hyperthermia upon microwave absorption and thereby dissolve aggregates of amyloid- $\beta .^{24,25}$ Nonetheless, AuNPs have not been investigated previously as potential enhancers or inhibitors of amyloid aggregation of AS. In this paper, we present a systematic study of the effects of AuNPs on the aggregation kinetics of AS, finding a noticeable dependency on the concentration as well as the size of the nanoparticles.

$\boldsymbol{\alpha}$-Synuclein Aggregation. The aggregation process of AS (Figure 1A) has been the subject of numerous investigations. ${ }^{1,26,27}$ On the way to the formation of amyloid fibrils, a variety of oligomeric species have been identified whose function or cytotoxicity remain uncertain. ${ }^{2,3,7,8,28,29}$ Macroscopically and in analogy to other amyloid proteins, the in vitro fibrillization of AS follows a kinetic profile typical of a nucleation-dependent polymerization process (Figure 1B) in which distinct phases of nucleation, propagation, and equilibration can be identified. ${ }^{26}$ The duration of the nucleation phase (also called lag-phase) as well as the rate of the propagation (also called growth phase) have been found to depend on several factors such as protein concentration, temperature, $\mathrm{pH}$, mechanical agitation, ionic strength, pointmutations in the amino acid sequence, and the presence of chaperone proteins. ${ }^{26,30-32}$

Initially, the system is conceived as undergoing nucleation in which pauci-oligomeric protein aggregates form and dissociate rapidly in accordance with the enthalpic and entropic balance of the interaction processes involved, a particularly relevant consideration in view of the largely unstructured nature of the AS monomer. ${ }^{33}$ Ultimately, structures (nuclei) arise with 

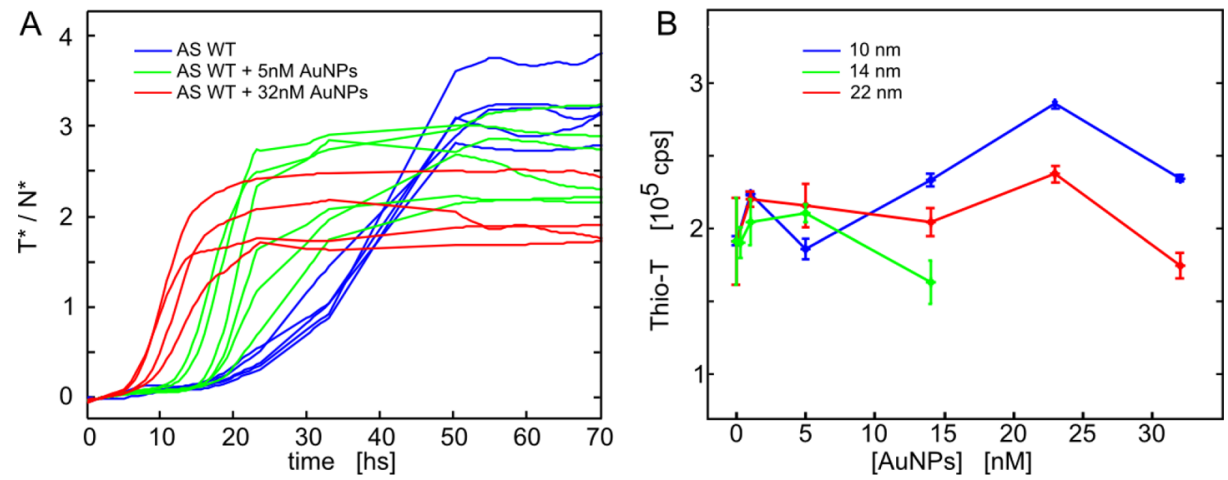

Figure 2. Monitoring the aggregation of AS in the presence of AuNPs. (A) Time trace of the ratio of emission bands $T^{*} / N^{*}$ for aggregation assays of AS wild type (WT) alone and in the presence of 5 and $32 \mathrm{nM}$ of AuNPs with a diameter of $14 \mathrm{~nm}$. (B) Average results of the ThioT measurement at the end of the aggregation assays performed with the three different AuNPs and concentrations ranging from 0 to $32 \mathrm{nM}$.

the capacity of promoting the stable addition of monomer units, at which point ordered polymerization proceeds rapidly. During this growth phase, the fraction of fibrillar product increases exponentially until a state of equilibrium between fibrils and monomers is achieved.

Monitoring the Aggregation of $\alpha$-Synuclein. There are several methods for the in vitro monitoring of amyloid protein aggregation $^{35}$ of which the most widely used is the increment in thioflavin-T(ThioT) fluorescence emission upon binding to the characteristic cross- $\beta$-sheet structure of the amyloid fibrils. ${ }^{36}$ We used the ThioT assay to quantify the amount of AS fibrils formed at the end of each aggregation experiment.

For the monitoring of the aggregation process in real time, a highly environment-sensitive fluorescent probe (MFC) was used. MFC exhibits dual emissions states: the "normal" excited state $\left(N^{*}\right)$ and a phototautomeric excited state $\left(T^{*}\right)$ product of intramolecular proton transfer (ESIPT). ${ }^{34}$ The MFC moiety (Figure 1C) was covalently linked to a monocysteine A140C mutant of AS its C-terminus. This labeled protein, denoted ASA140C-MFC, is a highly sensitive reporter for the aggregation of bulk AS. ${ }^{34}$ When AS-A140C-MFC is incorporated into oligomeric and fibrillar structures, the ESIPT probe senses a decrease in polarity, manifested by an increase in the $T^{*}$ emission (Figure 1C) and a corresponding change in the ratio of band intensities $\left(T^{*} / N^{*}\right)$. The aggregation assays monitored by MFC were performed with mixture of wild-type protein and $2 \%$ of AS-A140C-MFC. Under these conditions, parallel measurements of the $T^{*} / N^{*}$ intensity ratio of MFC and ThioT indicated that MFC is considerably more sensitive in the detection of the early stages of the aggregation., ${ }^{7,34}$ Furthermore, the ratiometric measurement of $T^{*} / N^{*}$ provides additional reliability in comparison to single intensity measurements (such as in the ThioT assay) because it is not affected by concentration differences nor by the eventual (photo-) degradation of the fluorescent probe during the extended time of the aggregation experiment. In addition, the method enables control experiments for evaluating the influence of absorption and quenching by the AuNPs (see Materials and Methods).

The effects of AuNPs on the process of aggregation were assessed by performing multiple replicate measurements in the presence of various concentrations of AuNPs of different sizes. The determinations were carried out with a 96-well microplate reader at $37{ }^{\circ} \mathrm{C}$, under constant shaking, and a total AS concentration of $100 \mu \mathrm{M}$ (98\% of AS wild type $+2 \%$ AS$\mathrm{A} 140 \mathrm{C}-\mathrm{MFC}$ ) in $10 \mathrm{mM}$ Na-citrate buffer $\mathrm{pH} 6.2$ and $0.2 \%$
$\mathrm{NaN}_{3}$. Citrate-capped AuNPs of three diameters $(10 \pm 2,14 \pm$ 2 , and $22 \pm 2 \mathrm{~nm}$ ) were added to the AS solution in concentrations ranging from 0.3 to $32 \mathrm{nM}$. MFC was excited at $340 \pm 10 \mathrm{~nm}$ and its emission bands were monitored at $420 \pm$ $10 \mathrm{~nm}\left(N^{*}\right)$ and $530 \pm 10 \mathrm{~nm}\left(T^{*}\right)$ (Figure 1C). The time course of the $T^{*} / N^{*}$ ratio provided a measure of the kinetics of aggregation (Figure 2A). The curves obtained presented the typical sigmoidal shape characteristic of the kinetics of a nucleation-dependent reaction in which the lag, growth, and equilibrium phases can be easily identified.

Effect of Gold Nanoparticles on the Aggregation Kinetics. The time courses of $T^{*} / N^{*}$ in replicate aggregation assays of AS in the presence of $10 \mathrm{~nm}$ diameter AuNPs at $5 \mathrm{nM}$ and $32 \mathrm{nM}$ concentrations are shown in Figure 2A, together with the results obtained for AS alone. The aggregation assays carried out in the presence of AuNPs exhibited the same sigmoidal profile characteristic of amyloid fibrillization observed in the absence of AuNPs (Figure 2A). In all cases a steady plateau value was achieved by $60 \mathrm{hs}$ of reaction time. The general effect of AuNPs was to strongly accelerate the reaction, clearly evidenced by the shift of the $T^{*} / N^{*}$ transition curves toward shorter times.

Another clear observation was that the final equilibrium $\mathrm{T}^{*} /$ $\mathrm{N}^{*}$ value diminished as the AuNP concentration increased. Although this could seem to indicate a lower yield of product fibrils, such was not the case. ThioT assays performed at the end of each aggregation assay showed that similar levels of fibrils were produced, that is, independent of the AuNP concentration (Figure 2B). Additional control experiments revealed that the reduction on $T^{*} / N^{*}$ was due to absorption and quenching of the MFC fluorescence by the AuNPs. The absorption and scattering of the AuNPs are dominated by a surface plasmon resonance band centered at about $530 \mathrm{~nm} .^{37}$ The $T^{*}$ band of MFC $(540 \mathrm{~nm})$ is more affected by the presence of the AuNPs than the $N^{*}$ band, thus reducing the values of $T^{*} / N^{*}$. In order to compare the kinetics, each curve of $T^{*} / N^{*}$ was normalized by its final constant value.

With the aim of quantifying the effect of the AuNPs on the aggregation kinetics, the curves of $T^{*} / N^{*}$ versus time were analyzed according to the Finke-Watzky (F-W) two-step model. ${ }^{38}$ According to this formalism, the many steps involved in the aggregation process at the molecular level are combined into two pseudoelementary steps that represent a slow nucleation and fast autocatalytic growth. ${ }^{38}$ This simple model oversimplifies the underlying aggregation process but has been demonstrated to account for a whole range of protein 

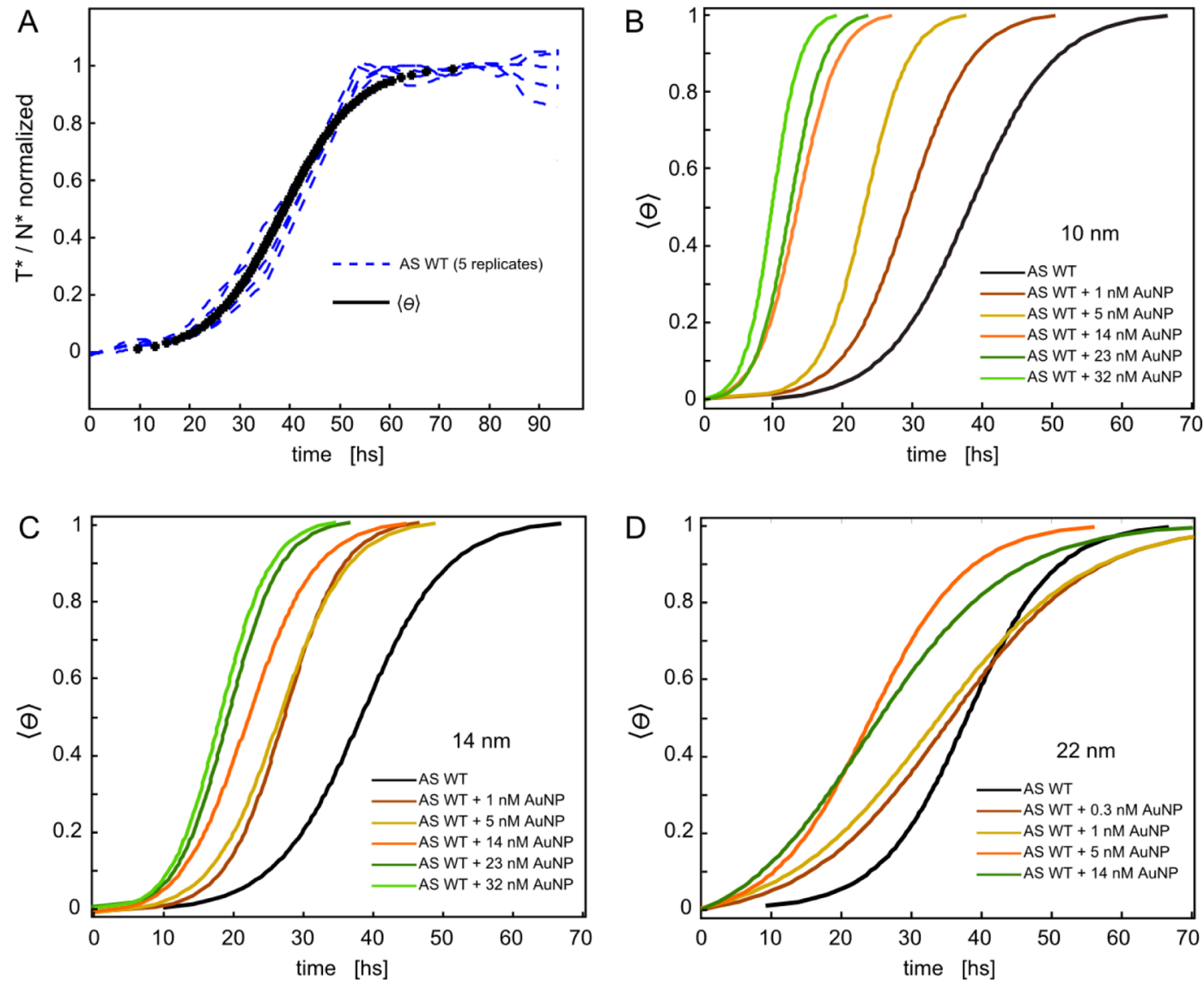

Figure 3. (A) Normalized $T^{*} / N^{*}$ ratio versus time obtained from five replicate measurements of the aggregation of ASWT (blue) and their average $\theta(t)$ (red). (B-D) Average $\theta(t)$ for samples with different concentrations of AuNPs with diameters 10, 14, and $22 \mathrm{~nm}$, respectively.

aggregation kinetic data. ${ }^{38,39}$ The F-W model, predicts that the fractional concentration of product as a function of time $\theta(t)$ is a sigmoidal function of only two independent parameters. Typical combinations of the two independent parameters are (i) the rates of the two pseudoelementary steps $k_{1}$ and $k_{2}$, or (ii) the time required to produce half the total product $t_{1 / 2}$ and the rate of growth at that time $v=\theta\left(t_{1 / 2}\right)$. We chose the latter representation because the given parameters are more easily visualized in the curves of the time course of aggregation. The fractional concentration of product $\theta(t)$ as a function of $t_{1 / 2}$ and $v$ is given as

$$
\theta(t)=\frac{1}{1+e^{-4 v\left(t-t_{1 / 2}\right)}}
$$

Another useful parameter to compare the kinetics is

$$
t_{N}=t_{1 / 2}-\frac{1}{2 v}
$$

Although $t_{N}$ is not independent (it is defined by $t_{1 / 2}$ and $v$ ), it facilitates the analysis of the influence of the AuNPs because it is a measure of the duration of the nucleation phase.

Each curve of $T^{*} / N^{*}$ was normalized by its final value and then fitted to eq 1 . In order to obtain a representative mean curve of $\theta(t)$ for each set of experimental conditions (AuNP size and concentration), the values of $t_{1 / 2}$ and $v$ obtained from the fit of an individual replicate measurement was used to generate an inverted function $t(\theta)$. The curves obtained in this manner for a given set of conditions were then averaged and inverted back. An average curve $\langle\theta(t)\rangle$ together with the raw data of the replicate aggregation curves for AS without AuNPs (WT) is shown in Figure 3A to illustrate the result of this procedure. The mean values obtained for the nucleation and propagation rates were $t_{1 / 2}=38 \pm 1 \mathrm{~h}$ and $v=0.041 \pm 0.01$ $\mathrm{h}^{-1}$, respectively for WT. The average curves $\langle\theta(t)\rangle$ of AS aggregation in the presence of AuNPs at different concentrations are shown in Figure 3B-D for the nanoparticles of 10, 14 , and $22 \mathrm{~nm}$ diameter, respectively. The mean curve for AS in the absence of nanoparticles is shown for comparison. A substantial acceleration of the aggregation as a function of the AuNP concentration was observed for the three diameters, the smaller AuNPs exerting the greatest effect.

The average half-time $t_{1 / 2}$ provides a measure of the overall effect of the AuNPs. For all diameters of AuNPs the average $t_{1 / 2}$ showed a progressive decrease as a function of AuNP concentration, arriving at a plateau at $\sim 20 \mathrm{nM}$ for the 10 and $14 \mathrm{~nm}$ AuNPs and at $5 \mathrm{nM}$ for the $22 \mathrm{~nm}$ AuNPs (Figure 4A). The maximum effect produced by the AuNPs of 10, 14, and 22 $\mathrm{nm}$ led to values of $t_{1 / 2}$ equal to 30,50 , and $75 \%$, of the value for AS alone, respectively; that is, the aggregation process was on the average 3,2 , and 1.5 times faster, compared to AS alone.

The overall acceleration produced by AuNPs can be attributed to distinct effects on the nucleation and growth phases of the aggregation. For the smaller AuNPs (10 and 14 $\mathrm{nm})$, there was a substantial increase in the rate of growth $v$ versus AuNP concentration (Figure 4B). At the highest concentrations, $v$ achieved values that were $\sim 2.5-3$ times higher than the growth rate of AS alone. In contrast, the larger AuNPs of $22 \mathrm{~nm}$ produced a weak reduction in $v$ of $\sim 40 \%$. 

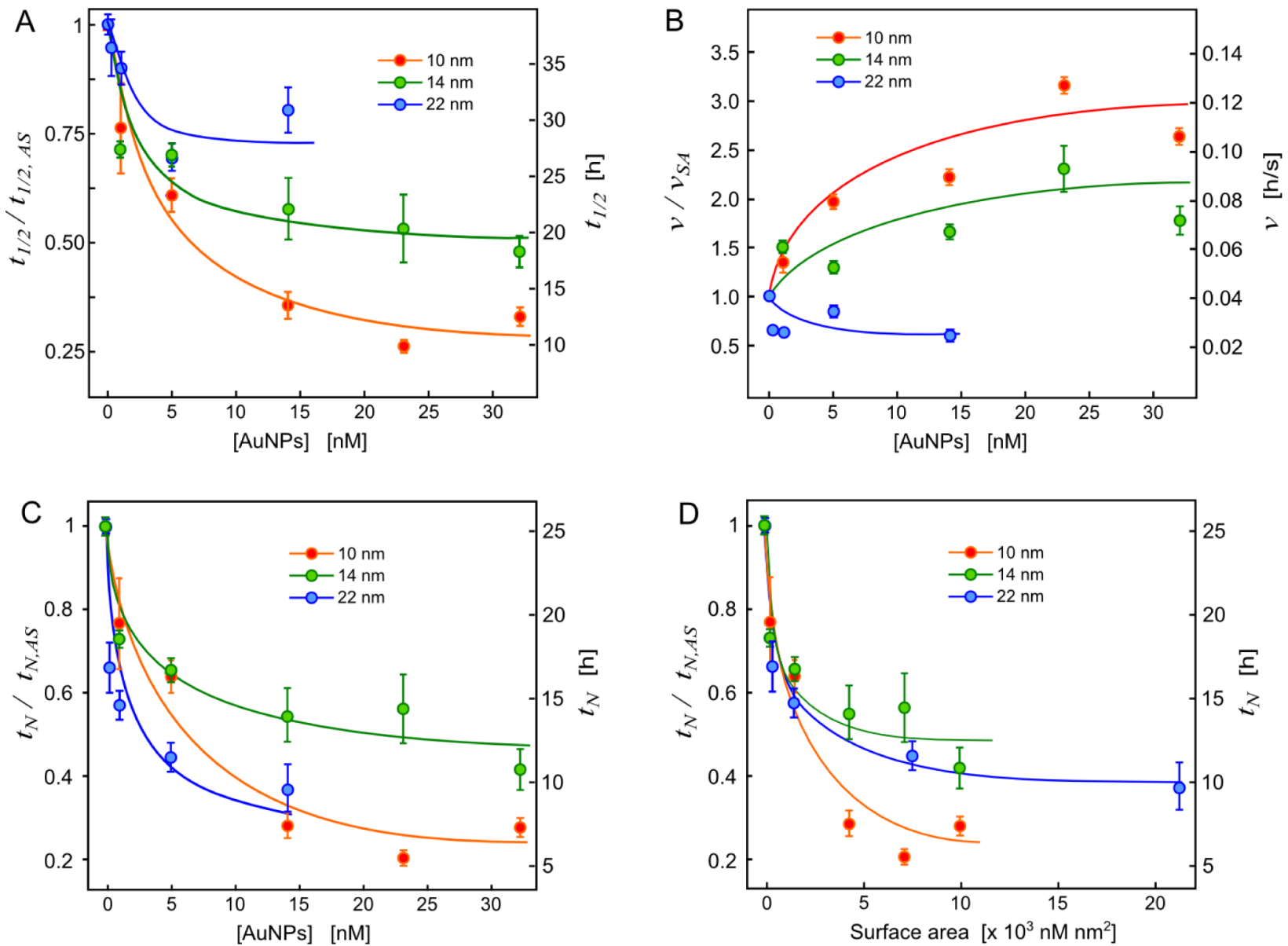

Figure 4. Dependence of the kinetic parameters on AuNP concentration and surface area. Half time (A), growth rate (B), and lag time (C), as a function of AuNP concentration for AuNP diameters of 10, 14, and $22 \mathrm{~nm}$. Lines are guides to the eye. (D) Growth rate as a function of AuNP surface area.

The AuNPs also produced a concentration dependent reduction of the duration of the lag phase $t_{\mathrm{N}}$ (Figure $4 \mathrm{C}$ ); the $22 \mathrm{~nm}$ AuNPs displayed the greatest effect. Already at the minimum concentration of $0.3 \mathrm{nM}$, the $22 \mathrm{~nm}$ AuNPs produced such a strong reduction of the lag time that growth was observed almost from the beginning of the aggregation assay (Figure 3D). Higher concentrations of $\sim 5 \mathrm{nM}$ were necessary to produce a similar facilitation of nucleation with the AuNPs of 14 and $10 \mathrm{~nm}$ (Figure 4C). We also addressed the question as to whether the effects on $t_{1 / 2}, v$, and $t_{\mathrm{N}}$ scaled with the surface area of AuNPs. In fact, for the lowest concentrations, the duration of the nucleation phase $t_{\mathrm{N}}$ indeed depended only on the amount of gold surface available and not on the size of the AuNPs (Figure 4D). At higher concentrations, the curves of $t_{\mathrm{N}}$ versus surface area for all AuNPs diverged from a linear behavior, becoming a more complex function of AuNP size. The rate of growth $v$ and $t_{1 / 2}$ did not scale with the surface area in any concentration range, indicating that the influence of the AuNPs cannot be explained as an effect due solely to the exposed surface.

Interaction of Gold Nanoparticles with the $\alpha$ Synuclein Fibrils. The fibrillar end products of aggregation were visualized by scanning electron microscope (SEM) (Figure 5A-D). No perceptible effect of the AuNPs on the morphology of the AS fibrils was detected. In all cases, typical AS fibrils with diameters of about $10-15 \mathrm{~nm}$ and lengths varying from a few hundreds of nanometers to a few micrometers were observed, independently of the concentration and size of the AuNPs.

From a visual inspection of the SEM images, a distinct affinity of the AuNPs to the AS fibrils was detected. The smaller AuNPs of 10 and $14 \mathrm{~nm}$ in diameter appeared distributed over the sample surface with no apparent affinity for the AS fibrils (Figure 5B,C). In contrast, the larger AuNPs of $22 \mathrm{~nm}$ were located more frequently over AS fibrils (Figures 5D). This observation was confirmed by a quantitative image analysis. Taking advantage of the markedly different electronic contrasts of $\mathrm{AS}$ and $\mathrm{Au}$, the AS fibrils and the AuNPs were identified using a dual threshold. Then for each SEM image the surface concentration of AuNPs was computed on the fibers $\left(C_{\mathrm{AuNP}, \mathrm{F}}\right)$ and on the rest of the sample surface $\left(C_{\mathrm{AuNP}}\right)$ as

$$
C_{\mathrm{AuNP}, \mathrm{F}}=\frac{N_{\mathrm{AuNP}, \mathrm{F}}}{A_{\mathrm{F}}} \quad C_{\mathrm{AuNP}}=\frac{\left(N_{\mathrm{AuNP}}-N_{\mathrm{AuNP}, \mathrm{F}}\right)}{\left(A-A_{\mathrm{F}}\right)}
$$

where $N_{\mathrm{AuNP}, \mathrm{F}}$ is the number of AuNPs found on top AS fibrils, $N_{\text {AuNP }}$ is the total number of AuNPs, $A_{\mathrm{F}}$ is the area of the sample covered with AS fibrils, and $A$ is the total area of the sample that was evaluated.

The average ratio of $C_{\mathrm{AuNP}, \mathrm{F}} / C_{\mathrm{AuNP}}$ for the end product of aggregation obtained with the AuNPs is shown in Figure 5D for the three diameters of AuNPs. A size-dependent affinity of the AuNPs for the AS fibrils is clearly evident. Both the 10 and 14 $\mathrm{nm}$ had a ratio of $C_{\mathrm{AuNP}, \mathrm{F}} / C_{\mathrm{AuNP}}$ smaller than 1 , indicating that 

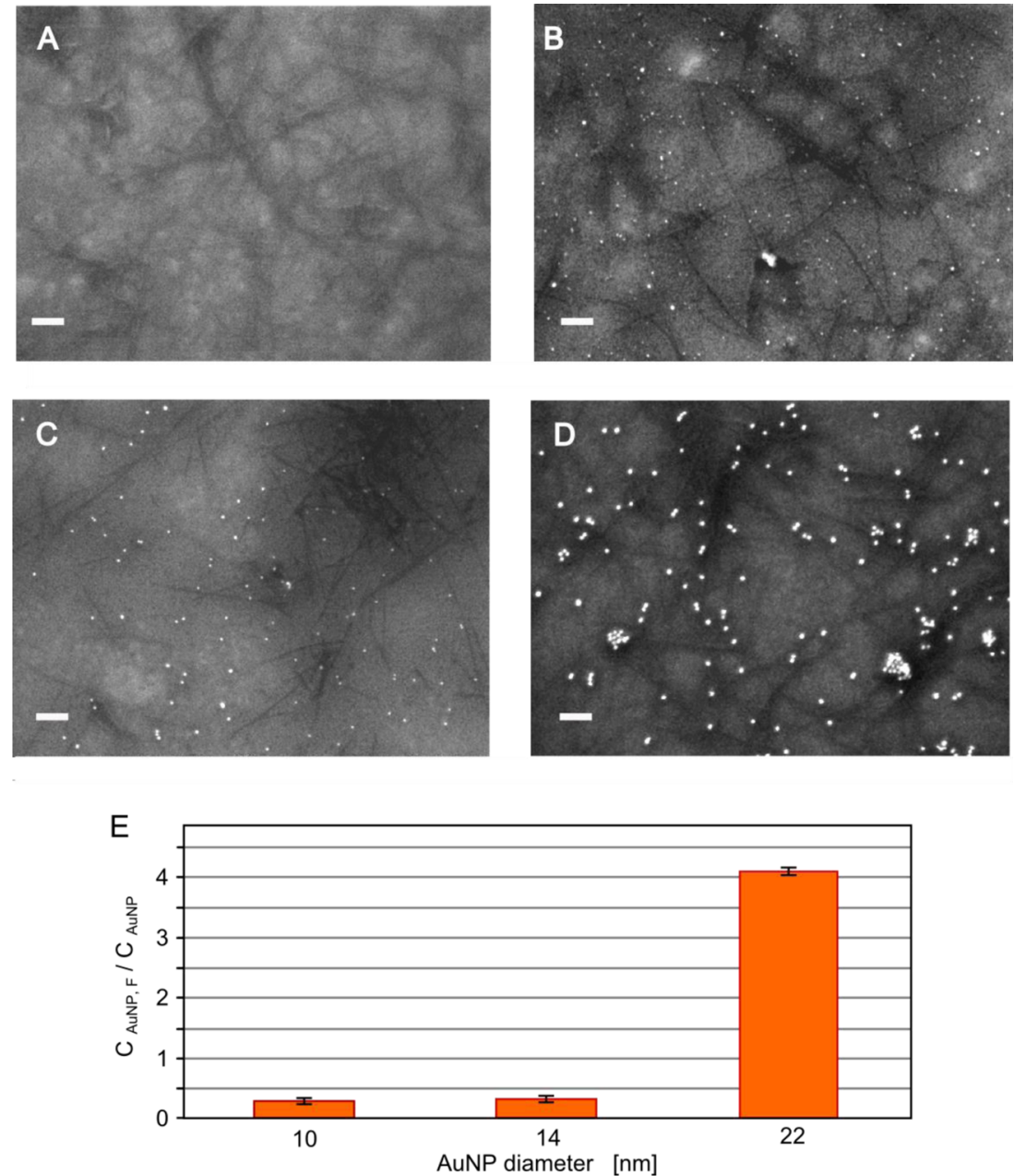

Figure 5. Scanning electron microscopy (SEM) characterization of the aggregation end product. (A-D) SEM images of the end product of the aggregation assay of AS WT alone (A), and with $14 \mathrm{nM}$ of AuNPs of $10 \mathrm{~nm}$ (B), $14 \mathrm{~nm}$ (C), and $22 \mathrm{~nm}$ (D) diameters. Scale bars $100 \mathrm{~nm}$. (E) Ratio of the surface concentrations of AuNPs observed on top of the AS fibrils $\left(C_{\mathrm{AuNP}, \mathrm{F}}\right)$ and on the rest of the sample $\left(C_{\mathrm{AuNP}}\right)$.

those smaller AuNPs remain preferentially unattached to the fibrils. In contrast, the surface concentration of $22 \mathrm{~nm}$ AuNPs bound to fibrils was $\sim 4$ times the surface concentration of free AuNPs. It should be noted that the three sets of AuNPs had the same surface capping with citrate. We conclude that the markedly greater affinity of the $22 \mathrm{~nm}$ for the AS fibrils is attributable primarily to the nanoparticle size and/or surface curvature.

Conclusions. This systematic study clearly shows that nanomolar concentrations of citrate-capped AuNPs produce a strong acceleration of the overall aggregation process of AS, an effect that is evident in the shortening of the half-time $t_{1 / 2}$. A more detailed analysis of the kinetics shows a distinct influence of the AuNPs on the duration of the nucleation phase $t_{\mathrm{N}}$ and the speed of propagation $v$. The shortening of $t_{\mathrm{N}}$ is indicative of an accumulation of protein on the nanoparticle surface. The local increased concentration of AS favors oligomerization and therefore the likelihood of formation of critical nuclei for fibrillization. It is found that for the lowest concentrations the duration of the nucleation phase depends only on the available gold surface. At higher concentrations, the influence of the AuNPs on $t_{\mathrm{N}}$ is more complex.

Second, it is found that the growth rate $v$ decreases as the nanoparticle diameter increases. Moreover, the smaller AuNPs (10 and $14 \mathrm{~nm}$ ) produce a strong acceleration of the growth rate whereas the larger $22 \mathrm{~nm}$ AuNPs inhibited growth somewhat. This result implies the AuNPs play a multifaceted role in the processes of aggregation and fibril formation, which goes beyond the local concentration increase responsible of the faster nucleation. In general, the interaction of proteins with nanoparticles is complex. For example, a systematic study of the interaction of lysozyme with silica nanoparticles showed that the native-like structure and function of the enzyme is better preserved upon absorption on smaller nanoparticles; adsorption on larger nanoparticles led to a reduction of $\alpha$-helical content and lower enzymatic activity. ${ }^{40}$ Similarly, a greater decrease in thermodynamic stability of RNase A was observed when the protein was adsorbed onto larger silica nanoparticles. ${ }^{41}$ As indicated earlier, the binding affinity of AS for citrate-capped gold nanoparticles of 20 and $90 \mathrm{~nm}$ is markedly higher for the larger nanoparticles. ${ }^{22}$ It is therefore reasonable to expect different protein conformations and structures upon adsorption onto nanoparticles of different sizes, in particular in the size range explored in this paper where the surface curvature varies significantly with respect to protein dimensions. ${ }^{42,43}$ The variations in growth rate could be attributed to the formation of different nuclei depending on the size of the nanoparticles, each of which then promotes growth of amyloid fibrils at different rates. 
In summary, we have demonstrated that gold nanoparticles can be used as efficient artificial chaperones to accelerate $\alpha$ synuclein aggregation. The strong catalytic effect of the gold nanoparticles is dependent on size and concentration. It is remarkable that the observed effects are noticeable at very low (nM) AuNP concentrations, facilitating potential cellular and animal experimentation for the investigation of potential therapeutic effects. These results encourage further investigations to elucidate the mechanistic details of the catalytic effect and exploration of the use of gold nanoparticles for the manipulation of the kinetics of aggregation of $\alpha$-synuclein and other amyloid proteins, for example with AuNPs functionalized with protein mutants.

Materials and Methods. $\alpha$-Synuclein (AS) Purification. A pT7-7 plasmids encoding the human AS sequences were expressed in E. coli BL21 (DE3) cells. Protein expression was induced with $1 \mathrm{mM}$ isopropyl- $\beta$-d-thiogalactopyranoside (IPTG) at $37{ }^{\circ} \mathrm{C}$, followed by alkaline lysis, boiling, and streptomycin sulfate $(10 \mathrm{mg} / \mathrm{mL})$ treatment. AS was precipitated from the supernatant by addition of solid $\left(\mathrm{NH}_{4}\right)_{2} \mathrm{SO}_{4}$ to a final concentration of $361 \mathrm{mg} / \mathrm{mL}$. The pellets containing AS were stored at $-20{ }^{\circ} \mathrm{C}$. Purification of AS was carried out with a GE Healthcare ÄKTA explorer HPLC system with an Applied Biosystems POROS HQ $20 \mu \mathrm{m}, 4.6 \times$ $100 \mathrm{~mm}(1.7 \mathrm{~mL})$ column. Samples were dialyzed in $10 \mathrm{mM}$ sodium citrate $\mathrm{pH} 6.2$ and concentrated with Millipore Amicon $10 \mathrm{kDa}$ filters, frozen with liquid $\mathrm{N}_{2}$, and storage at $-80{ }^{\circ} \mathrm{C}$. The concentrations of AS solutions were determined by measuring the UV absorbance at $275 \mathrm{~nm}$ using an extinction coefficient of $5200 \mathrm{M}^{-1} \mathrm{~cm}^{-1}$. In order to achieve reproducible conditions in the aggregation assays, ultracentrifugation was performed $(1 \mathrm{~h}$ at $105000 \times g)$ in a Beckman 80 TLS-55 rotor before each experiment.

MFC Labeling. MFC was synthesized according to Yushchenko et al. ${ }^{34}$ For the labeling procedure, MFC was mixed with a solution of $300 \mu \mathrm{M}$ of a A140C mutant of AS (AS-A140C) in $25 \mathrm{mM} \mathrm{Na}-\mathrm{PO}_{4} \mathrm{pH} 7.3,3 \mathrm{mM}$ TCEP; in a 510 -fold molar excess over the protein. The mixture was left stirring at $4{ }^{\circ} \mathrm{C}$ overnight. A size exclusion PD-10 column was then used as a first step of purification from the unbound dye, followed by chromatography system with a Sepharose G15 column in $25 \mathrm{mM}$ Tris-HCl, $\mathrm{pH}$ 7.2. Samples were frozen in liquid $\mathrm{N}_{2}$ and stored at $-80{ }^{\circ} \mathrm{C}$.

Aggregation Assay. The aggregation assay was performed on a microplate reader (Pherastar BMG-Labtech). Fluorescence was excited at $340 \pm 10 \mathrm{~nm}$, and emission collected with dual emission filters at $420 \pm 10 \mathrm{~nm}\left(N^{*}\right.$ band $)$ and $540 \pm 10$ $\mathrm{nm}\left(T^{*}\right.$ band). The fluorescence was measured every $30 \mathrm{~min}$. Samples containing $150 \mu \mathrm{L}$ of $100 \mu \mathrm{M}$ AS, $2 \%$ of AS-A140CMFC in $10 \mathrm{mM}$ citrate buffer $\mathrm{pH} 6.2$ with $0.2 \% \mathrm{NaN} 3$ was incubated on 96 wells plates at $37{ }^{\circ} \mathrm{C}$ with double orbital 500 rpm shaking. Gold nanoparticles at different concentrations between 0.3 and $32 \mathrm{nM}$ were added to the AS solutions.

Control Experiments. Aggregation assays of AS WT under identical buffer conditions (10 mM Na-citrate, $\mathrm{pH} 6.2$, and $0.2 \% \mathrm{NaN}_{3}$ ), protein concentration (total AS concentration of $100 \mu \mathrm{M}$ with $98 \%$ of AS wild type $+2 \%$ AS-A140C-MFC) and temperature $\left(37^{\circ} \mathrm{C}\right)$ as described above were carried out but in this case a larger volume of $800 \mu l$ was used in a cuvette and the fluorescence emission spectrum of MFC was monitored in a spectrofluorometer (Varian Cary Eclipse, Agilent Technolgies) instead of the microplate reader with excitation at $340 \pm 10 \mathrm{~nm}$. Instead of shaking, magnetic stirring was applied. Aliquots of 80 $\mu \mathrm{L}$ were extracted at different aggregation times of $0,14,22,36$, and $42 \mathrm{~h}$. The aliquots were divided in four samples of $20 \mu \mathrm{L}$ and subsequently mixed with AuNP colloids so as to achieve AuNPs of concentrations of 5, 14, 23, and $32 \mathrm{nM}$ as used in the other measurements. The final concentration of AS was somewhat different in each case but the ratiometric measurement of $T^{*} / N^{*}$ does not depend on the MFC concentration. The $T^{*} / N^{*}$ ratio was measured for all samples in 386-well plates in the microplate reader under the same conditions used for the aggregation assays. The measured values of $T^{*} / N^{*}$ decreased in proportion to the concentration of AuNPs but kinetics were not affected significantly.

Gold Nanoparticles. Nanoparticles with three different diameters were used. AuNPs with a $10 \mathrm{~nm}$ diameter were synthetized with $\mathrm{NaBH}_{4}{ }^{44}$ the $14 \mathrm{~nm}$ diameter AuNPs were synthetized by the Turkevich method, ${ }^{45}$ and the $20 \mathrm{~nm}$ diameter AuNPs were purchased from Nanopartz (A11-20). For synthesis of AuNP with $\mathrm{NaBH}_{4}, 600 \mu \mathrm{L}$ of an aqueous stock solution of $50 \mathrm{mM} \mathrm{NaBH}_{4}, 50 \mathrm{mM} \mathrm{NaOH}$ was added to $100 \mu \mathrm{L}$ of an aqueous stock solution of $50 \mathrm{mM}$ ionized gold trichloride hydrochloride $\left(\mathrm{HAuCl}_{4}\right)$ at room temperature. After $10 \mathrm{~min}$ stirring, the solution was heated for 2-3 $\mathrm{min}$ in boiling water. In the Turkevich method, $47.5 \mathrm{~mL}$ of $0.5 \mathrm{mM} \mathrm{HAuCl}$ (ultrapure water) was heated to boiling in a $250 \mathrm{~mL}$ Erlenmeyer flask. Sodium citrate $(1 \% \mathrm{w} / \mathrm{w}, 2.5 \mathrm{~mL})$ was added, and the solution allowed to boil for another $30 \mathrm{~min}$. The AuNPs were washed by several steps of centrifugation and resuspended in $10 \mathrm{mM}$ citrate, $\mathrm{pH}$ 6.2. The AuNP diameters were determined by analysis of scanning electron microscopy (SEM) images with ImageJ. The extinction coefficients of nanoparticles were obtained from Mie theory and the concentrations were determined by UV-vis spectroscopy in a spectrophotometer (Varian Eclispe).

ThioT Assay. Ten microliter aggregation samples were diluted in $10 \mathrm{~mL}$ of $5 \mu \mathrm{M}$ ThioT in $50 \mathrm{mM}$ glycine buffer $\mathrm{pH}$ 8.2. After $15 \mathrm{~min}$ of shaking, 6 replicates from each sample were transferred to a 96-well plate. Samples were excited at $420 \pm 10$ $\mathrm{nm}$ and emission was measured at $460 \pm 10 \mathrm{~nm}$.

SEM Images. SEM images were taken with FEG-SEM SUPRA 40 (Carl Zeiss NTS) at $2 \mathrm{keV}$, in the Centro de Microscopías Avanzadas (CMA), Facultad de Ciencias Exactas y Naturales, Universidad de Buenos Aires.

\section{AUTHOR INFORMATION}

\section{Corresponding Author}

*E-mail: fernando.stefani@df.uba.ar.

\section{Author Contributions}

"Y.D.A. and J.A.F. contributed equally.

\section{Notes}

The authors declare no competing financial interest.

${ }^{\perp}$ Deceased on September 29th, 2011.

\section{ACKNOWLEDGMENTS}

This study was supported by the Max Planck Society (MPG) through the E.J.-E., Partner Group grant, Argentine agencies: Agencia Nacional de Promoción Científica y Tecnológica (ANPCYT), Consejo Nacional de Investigaciones Científicas y Técnicas (CONICET) and Universidad de Buenos Aires, as well as the joint grant MPG-ANPCYT, Toxic Protein Conformation project PICT 2010-2817 (T.M.J and E.J.-E). 


\section{REFERENCES}

(1) Breydo, L.; Wu, J. W.; Uversky, V. N. $\alpha$-synuclein misfolding and Parkinson's disease. Biochim. Biophys. Acta 2012, 1822, 261-85.

(2) Lansbury, P. T.; Lashuel, H. A. Review Article A century-old debate on protein aggregation and neurodegeneration enters the clinic. Nature 2006, 443, 774-779.

(3) Winner, B.; et al. In vivo demonstration that alpha-synuclein oligomers are toxic. Proc. Natl. Acad. Sci. U.S.A. 2011, 108, 4194-9.

(4) Steiner, J. A; Angot, E.; Brundin, P. A deadly spread: cellular mechanisms of $\alpha$-synuclein transfer. Cell Death Differ. 2011, 18, 142533.

(5) Luk, K. C.; et al. Pathological $\alpha$-synuclein transmission initiates Parkinson-like neurodegeneration in nontransgenic mice. Science 2012, 338, 949-53.

(6) Pornsuwan, S.; et al. Long-Range Distances in Amyloid Fibrils of $\alpha$-Synuclein from PELDOR Spectroscopy. Angew. Chem. 2013, DOI: 10.1002 /ange. 201304747.

(7) Fauerbach, J. A.; et al. Supramolecular non-amyloid intermediates in the early stages of $\alpha$-synuclein aggregation. Biophys. J. 2012, 102, 1127-36.

(8) Roberti, M. J.; et al. Imaging nanometer-sized $\alpha$-synuclein aggregates by superresolution fluorescence localization microscopy. Biophys. J. 2012, 102, 1598-607.

(9) Linse, S.; et al. Nucleation of protein fibrillation by nanoparticles. Proc. Natl. Acad. Sci. U.S.A. 2007, 104, 8691-6.

(10) Cabaleiro-Lago, C.; Szczepankiewicz, O.; Linse, S. The Effect of Nanoparticles on Amyloid Aggregation Depends on the Protein Stability and Intrinsic Aggregation Rate. Langmuir 2012, 28, 1852-7.

(11) Cabaleiro-Lago, C.; Quinlan-Pluck, F.; Lynch, I.; Dawson, K. A.; Linse, S. Dual Effect of Amino Modified Polystyrene Nanoparticles on Amyloid $\beta$ Protein Fibrillation. ACS Chem. Neurosci. 2010, 1, 279-87.

(12) Cabaleiro-Lago, C.; et al. Inhibition of Amyloid Beta Protein Fibrillation by Polymeric Nanoparticles. J. Am. Chem. Soc. 2008, 130, 15437-15443.

(13) Brambilla, D.; et al. PEGylated Nanoparticles Bind to and Alter Amyloid-Beta Peptide Conformation: Toward Engineering of Functional Nanomedicines for Alzheimer's Disease. ACS Nano 2012, 6, 5897-5908.

(14) Roberti, M. J.; et al. Quantum Dots as Ultrasensitive Nanoactuatorsand Sensors of Amyloid Aggregation in Live Cells. J. Am. Chem. Soc. 2009, 131, 8102-8107.

(15) Roberti, M. J.; Giordano, L.; Jovin, T. M.; Jares-Erijman, E. A. FRET imaging by $\mathrm{k}(\mathrm{t}) / \mathrm{k}(\mathrm{f})$. ChemPhysChem 2011, 12, 563-6.

(16) Jain, P. K.; Lee, K. S.; El-Sayad, I. H.; El-Sayed, M. A. Calculated Absorption and Scattering Properties of Gold Nanoparticles of Different Size, Shape, and Composition: Applications in Biological Imaging and Biomedicine. J. Phys. Chem. B 2006, 110, 7238.

(17) Chithrani, B. D.; Ghazani, A. A.; Chan, W. C. W. Determining the Size and Shape Dependence of Gold Nanoparticle Uptake into Mammalian Cells. Nano Lett. 2006, 6, 662-8.

(18) Shukla, R; et al. Biocompatibility of Gold Nanoparticles and Their Endocytotic Fate Inside the Cellular Compartment: A Microscopic Overview. Langmuir 2005, 21, 10644-54.

(19) Sperling, R. A.; Rivera Gil, P.; Zhang, F.; Zanella, M.; Parak, W. J. Biological applications of gold nanoparticles. Chem. Soc. Rev. 2008, 37, 1896-908.

(20) Murphy, C. J.; et al. Gold nanoparticles in biology: beyond toxicity to cellular imaging. Acc. Chem. Res. 2008, 41, 1721-30.

(21) Khlebtsov, N.; Dykman, L. Biodistribution and toxicity of engineered gold nanoparticles: a review of in vitro and in vivo studies. Chem. Soc. Rev. 2011, 40, 1647-71.

(22) Yang, J. A.; et al. Study of Wild-Type $\alpha$-Synuclein Binding and Orientation on Gold Nanoparticles. Langmuir 2013, 29, 4603-15.

(23) Ai Tran, H. N.; et al. A novel class of potential prion drugs: preliminary in vitro and in vivo data for multilayer coated gold nanoparticles. Nanoscale 2010, 2, 2724-32.

(24) Bastus, N. G.; et al. Gold nanoparticles for selective and remote heating of $\beta$-amyloid protein aggregates. Mater. Sci. Eng., C 2007, 27, $1236-1240$.
(25) Kogan, M. J.; et al. Nanoparticle-Mediated Local and Remote Manipulation of Protein Aggregation. Nano Lett. 2006, 6, 110-5.

(26) Fink, A. L. The Aggregation and Fibrillation of alpha-Synuclein. Acc. Chem. Res. 2006, 39, 628-634.

(27) Giehm, L.; Lorenzen, N.; Otzen, D. E. Assays for $\alpha$-synuclein aggregation. Methods 2011, 53, 295-305.

(28) Kaylor, J.; et al. Characterization of Oligomeric Intermediates in a-Synuclein Fibrillation: FRET Studies of Y125W/Y133F/Y136F alpha-Synuclein. J. Mol. Biol. 2005, 353, 357-372.

(29) Outeiro, T. F.; et al. Formation of toxic oligomeric alphasynuclein species in living cells. PLoS One 2008, 3, e1867.

(30) Morris, A. M.; Finke, R. G. Alpha-synuclein aggregation variable temperature and variable $\mathrm{pH}$ kinetic data: a re-analysis using the FinkeWatzky 2-step model of nucleation and autocatalytic growth. Biophys. Chem. 2009, 140, 9-15.

(31) Morris, A. M.; Watzky, M. A; Finke, R. G. Protein aggregation kinetics, mechanism, and curve-fitting: a review of the literature. Biochim. Biophys. Acta 2009, 1794, 375-97.

(32) Lee, C. C.; Nayak, A.; Sethuraman, A.; Belfort, G.; McRae, G. J. A three-stage kinetic model of amyloid fibrillation. Biophys. J. 2007, 92 (10), 3448-3458.

(33) Bertoncini, C. W.; et al. Release of long-range tertiary interactions potentiates aggregation of natively unstructured alphasynuclein. Proc. Natl. Acad. Sci. U.S.A. 2005, 102, 1430-5.

(34) Yushchenko, D. a; Fauerbach, J. A.; Thirunavukkuarasu, S.; Jares-erijman, E. a; Jovin, T. M. Fluorescent Ratiometric MFC Probe Sensitive to Early Stages of Alpha-Synuclein Aggregation. J. Am. Chem. Soc. 2010, 132, 7860-1.

(35) Bertoncini, C. W.; Celej, M. S. Small molecule fluorescent probes for the detection of amyloid self-assembly in vitro and in vivo. Curr. Protein Pept. Sci. 2011, 12, 205-20.

(36) Groenning, M. Binding mode of Thioflavin $T$ and other molecular probes in the context of amyloid fibrils-current status. J. Chem. Biol. 2010, 3, 1-18.

(37) Coronado, E. a; Encina, E. R.; Stefani, F. D. Optical properties of metallic nanoparticles: manipulating light, heat and forces at the nanoscale. Nanoscale 2011, 3, 4042-4059.

(38) Morris, A. M.; Watzky, M. A.; Agar, J. N.; Finke, R. G. Fitting neurological protein aggregation kinetic data via a 2-step, minimal/ "Ockham's razor" model: the Finke-Watzky mechanism of nucleation followed by autocatalytic surface growth. Biochemistry 2008, 47, 241327.

(39) Watzky, M. A; Morris, A. M.; Ross, E. D.; Finke, R. G. Fitting Yeast and Mammalian Prion Aggregation Kinetic Data with the FinkeWatzky Two-Step Model of Nucleation and Autocatalytic Growth. Biochemistry 2008, 47, 10790-800.

(40) Vertegel, A. a; Siegel, R. W.; Dordick, J. S. Silica Nanoparticle Size Influences the Structure and Enzymatic Activity of Adsorbed Lysozyme. Langmuir 2004, 20, 6800-7.

(41) Shang, W.; Nuffer, J. H.; Dordick, J. S.; Siegel, R. W. Unfolding of ribonuclease A on silica nanoparticle surfaces. Nano Lett. 2007, 7, 1991-5.

(42) Goy-López, S.; et al. Physicochemical Characteristics of ProteinNP Bioconjugates: The Role of Particle Curvature and Solution Conditions on Human Serum Albumin Conformation and Fibrillogenesis Inhibition. Langmuir 2012, 28, 9113-26.

(43) Fei, L.; Perrett, S. Effect of nanoparticles on protein folding and fibrillogenesis. Int. J. Mol. Sci. 2009, 10, 646-55.

(44) Martin, M. N.; Basham, J. I.; Chando, P.; Eah, S.-K. Charged Gold Nanoparticles in Non-Polar Solvents: 10-min Synthesis and 2D Self-Assembly. Langmuir 2010, 26, 7410-7.

(45) Kimling, J.; et al. Turkevich Method for Gold Nanoparticle Synthesis Revisited. J. Phys. Chem. B 2006, 110, 15700-15707. 